\title{
Stability results for second-order evolution equations with switching time-delay
}

\author{
Serge Nicaise \\ Institut des Sciences et Techniques de Valenciennes \\ Laboratoire de Mathématiques et leurs Applications \\ Université de Valenciennes et du Hainaut Cambrésis \\ 59313 Valenciennes Cedex 9, France \\ Cristina Pignotti \\ Dipartimento di Ingegneria e Scienze dell'Informazione e Matematica \\ Università di L'Aquila \\ Via Vetoio, Loc. Coppito, 67010 L'Aquila Italy
}

\begin{abstract}
We consider second-order evolution equations in an abstract setting with intermittently delayed/not-delayed damping. We give sufficient conditions for asymptotic and exponential stability, improving and generalising our previous results from [19. In particular, under suitable conditions, we can consider unbounded damping operators. Some concrete examples are finally presented.
\end{abstract}

2000 Mathematics Subject Classification: 35L05, 93D15

Keywords and Phrases: wave equation, delay feedbacks, stabilization

\section{Introduction}

Let $H$ be a real Hilbert space and let $A: \mathcal{D}(A) \rightarrow H$ be a positive self-adjoint operator with a compact inverse in $H$. Denote by $V:=\mathcal{D}\left(A^{\frac{1}{2}}\right)$ the domain of $A^{\frac{1}{2}}$. Moreover, for $i=1,2$, let $U_{i}$ be real Hilbert spaces with norm and inner product denoted respectively by $\|\cdot\|_{U_{i}}$ and $\langle\cdot, \cdot\rangle_{U_{i}}$ and let $B_{i}(t): U_{i} \rightarrow V^{\prime}$, be time-dependent linear operators satisfying

$$
B_{1}(t) B_{2}(t)=0, \quad \forall t>0 .
$$

Let us consider the problem

$$
\begin{aligned}
& u_{t t}(t)+A u(t)+B_{1}(t) B_{1}^{*}(t) u_{t}(t)+B_{2}(t) B_{2}^{*}(t) u_{t}(t-\tau)=0 \quad t>0 \\
& u(0)=u_{0} \quad \text { and } \quad u_{t}(0)=u_{1}
\end{aligned}
$$

where the constant $\tau>0$ is the time delay. We assume that the delay feedback operator $B_{2}$ is bounded, that is $B_{2} \in \mathcal{L}\left(U_{2}, H\right)$, while the standard one $B_{1} \in \mathcal{L}\left(U_{1}, V^{\prime}\right)$ may be unbounded. 
Time delay effects appear in many applications and practical problems and it is by now well-known that even an arbitrarily small delay in the feedback may destabilize a system wich is uniformly exponentially stable in absence of delay. For some examples of this destabilizing effect of time delays we refer to [4, [5, 17, 22].

In [17] we considered the wave equation with both dampings acting simultaneously, that is $B_{1}(t)=\mu_{1}$ and $B_{2}(t)=\mu_{2}$, with $\mu_{1}, \mu_{2} \in \mathbb{R}^{+}$, and we proved that if $\mu_{1}>\mu_{2}$ then the system is uniformly exponentially stable. Otherwise, if $\mu_{2} \geq \mu_{1}$, that is the delay term prevails on the not delayed one, then there are instability phenomena, namely there are unstable solutions for arbitrarily small (large) delays.

In [19] we firstly considered second-order evolution equations with intermittent delay, this means that the standard damping and the delayed one act in different time intervals. This is clearly related to the stabilization problem of second-order evolution equations damped by positive/negative feedbacks. We refer for this subject to [7]. See also [20] for the link between wave equation with time delay in the damping and wave equation with indefinite damping, namely damping which may change sign in different subsets of the domain.

Assuming that an observability inequality holds for the conservative model associated with (1.1) -(1.2) and, through the definition of a suitable energy (see (3.3)), we obtained in [19] sufficient conditions ensuring asymptotic stability. Under more restrictive assumptions an exponential stability estimate was also obtained. Our abstract framework was then applied to some concrete examples, namely the wave equation, the elasticity system and the Petrovsky system.

We mention that a similar problem has been considered in [2] for 1-d models for the wave equation but with a different approach. Indeed, in [2] we obtain stability results for particular values of the time delays, related to the length of the domain, by using the D'Alembert formula.

Here we improve the results of [19] removing a quite restrictive assumption on the size of the "bad" terms, that is the terms with time delay (cfr. assumption (3.3) of [19]). Indeed, as we expect from [7] and considering the relation between delay problems and problems with anti-damping, the delay feedback operator $B_{2}$ may be also large but on small time intervals. Moreover, under an additional assumption on the size of the time intervals where only the delay feedback acts, we give stability results also for $B_{1}$ unbounded, while the method in [19] is applicable only for bounded damping operators $B_{1}$. Some new examples, not covered by the analysis of [19], are illustrated. Furthermore, we point out the improvement for examples already considered there.

The paper is organized as follows. In section 2 a well-posedness result of the abstract system is proved. In section 3 we obtain, for $B_{1}$ bounded, asymptotic and exponential stability results for the abstract model under suitable conditions, improving the results of [19]. In section 4, under an additional condition on the length of the delay intervals, we obtain stability results valid for $B_{1}$ non necessarily bounded. Finally, in section 5 , we illustrate our abstract results by some concrete applications.

\section{Well-posedness}

In this section we will give well-posedness results for problem (1.1)-(1.2) using semigroup theory.

We assume that for all $n \in \mathbb{N}$, there exists $t_{n}>0$ with $t_{n}<t_{n+1}$ and such that

$$
B_{2}(t)=0 \forall t \in I_{2 n}=\left[t_{2 n}, t_{2 n+1}\right),
$$




$$
B_{1}(t)=0 \forall t \in I_{2 n+1}=\left[t_{2 n+1}, t_{2 n+2}\right),
$$

with $B_{2} \in C\left(\left[t_{2 n+1}, t_{2 n+2}\right] ; \mathcal{L}\left(U_{2}, H\right)\right)$; for the operators $B_{1}$, we assume either

$$
B_{1} \in C^{1}\left(\left[t_{2 n}, t_{2 n+1}\right] ; \mathcal{L}\left(U_{1}, H\right)\right)
$$

or

$$
B_{1}(t)=\sqrt{b_{1}(t)} C_{n}
$$

with $\left.C_{n} \in \mathcal{L}\left(U_{1}, V^{\prime}\right)\right)$ and $b_{1} \in W^{2, \infty}\left(t_{2 n}, t_{2 n+1}\right)$ such that

$$
b_{1}(t)>0, \forall t \in I_{2 n} .
$$

We further assume that $\tau \leq T_{2 n}$ for all $n \in \mathbb{N}$, where $T_{n}$ denotes the lengt of the interval $I_{n}$, that is

$$
T_{n}=t_{n+1}-t_{n}, \quad n \in \mathbb{N} .
$$

Under these assumptions, we obtain the following result

Theorem 2.1 Under the above assumptions, for any $u_{0} \in V$ and $u_{1} \in H$, the system (1.1) (1.2) has a unique solution $u \in C([0, \infty) ; V) \cap C^{1}([0, \infty) ; H)$.

Proof. The case $B_{1} \in C^{1}\left(\left[t_{2 n}, t_{2 n+1}\right] ; \mathcal{L}\left(U_{1}, H\right)\right)$ was treated in Theorem 2.1 of [19], hence we concentrate on the case when $B_{1}$ is not bounded. In view of the proof of Theorem 2.1 of [19] and the assumption on $B_{1}$, we only need to prove existence in the interval $\left(0, t_{1}\right)$ of the problem

$$
\begin{aligned}
& u_{t t}(t)+A u(t)+b_{1}(t) C_{1} C_{1}^{*} u_{t}(t)=0 \quad t>0 \\
& u(0)=u_{0} \quad \text { and } \quad u_{t}(0)=u_{1} .
\end{aligned}
$$

Here the difficulty is that a priori the domain of the operator

$$
\mathcal{A}(t)(u, v)^{\top}=\left(v,-A u-b_{1}(t) C_{1} C_{1}^{*} v\right)^{\top}
$$

will depend on $t$ since it requires that $A u+B_{1}(t) B_{1}^{*}(t) v$ has to be in $H$. Hence we cannot directly use the theory developed by Kato, see [8, 9]. proof)

The solution is to set (for shorthness the index 1 is suppressed in the remainder of the

$$
U=\left(u, b u_{t}\right)^{\top}
$$

that satisfies formally

$$
U_{t}=\mathcal{A}(t) U, U(0)=\left(u_{0}, b(0) u_{1}\right) \in V \times H
$$

where

$$
\mathcal{A}(t)(u, v)^{\top}=\left(b^{-1} v, b^{\prime} b^{-1} v-b A u-b C C^{*} v\right) .
$$

The main idea is that the domain of this new operator is independent of $t$, since it is given by

$$
D(\mathcal{A}(t))=\left\{(u, v)^{\top} \in V \times V: A u+C C^{*} v \in H\right\} .
$$

Now we introduce the time-depending inner product (on $V \times H$ )

$$
\left((u, v)^{\top},(\tilde{u}, \tilde{v})^{\top}\right)_{t}=b^{2}(t)\left(A^{1 / 2} u, A^{1 / 2} \tilde{u}\right)_{H}+(v, \tilde{v})_{H}, \quad \forall(u, v)^{\top},(\tilde{u}, \tilde{v})^{\top} \in V \times H,
$$


and let $\|U\|_{t}=(U, U)_{t}^{1 / 2}$ its associated norm. It is easy to check that (see for instance Theorem 2.3 of [18])

$$
\|U\|_{t} \leq\|U\|_{s} e^{\kappa|t-s|}, \forall t, s \in\left(0, t_{1}\right)
$$

where $\kappa=\max _{t \in\left[0, t_{1}\right]} \frac{\left|b^{\prime}(t)\right|}{b(t)}$.

It is a simple exercise to check that

$$
\tilde{\mathcal{A}}(t)=\mathcal{A}(t)-\kappa I d
$$

generates a $C_{0}$-semigroup of contraction on $V \times H$ (that is dissipative for inner product $(\cdot, \cdot)_{t}$ ). By the assumption on $b$, we deduce that $\tilde{\mathcal{A}}=\left\{\tilde{\mathcal{A}}(t) ; t \in\left[0, t_{1}\right]\right\}$ and $Y=D(\mathcal{A}(0))$ forms a CD-system (or constant domain system) in the sense of Kato, see [8, 9]. In other words, for all $U_{0}=\left(u_{0}, b(0) u_{1}\right) \in V \times H$ (resp. $\left.D(\mathcal{A}(0))\right)$, there exists a unique mild (resp. strong) solution $\tilde{U} \in C\left(\left[0, t_{1}\right] ; V \times H\right)\left(\operatorname{resp} . \tilde{U} \in C\left(\left[0, t_{1}\right] ; D(\mathcal{A}(0))\right) \cap C^{1}\left(\left[0, t_{1}\right] ; V \times H\right)\right)$ of

$$
\tilde{U}_{t}(t)=\tilde{\mathcal{A}}(t) \tilde{U}(t), t>0, \quad U(0)=U_{0} .
$$

Setting

$$
U(t)=e^{\kappa t} \tilde{U}(t),
$$

we deduce that it is a mild (resp. strong) solution of

$$
U_{t}(t)=\mathcal{A}(t) \tilde{U}(t), t>0, \quad U(0)=U_{0} .
$$

Coming back to the definition of $\mathcal{A}(t)$, we find that $u$ is a solution of (2.2) $-(2.3)$.

\section{Stability result: $B_{1}$ bounded}

In this section we assume $B_{1}$ bounded. To get stability we assume that there exist Hilbert spaces $W_{i}, i=1,2$, such that $H$ is continuously embedded into $W_{i}$, i.e.

$$
\|u\|_{W_{i}}^{2} \leq C_{i}\|u\|_{H}^{2}, \quad \forall u \in H \text { with } C_{i}>0 \text { independent of } u .
$$

Moreover, we assume that for all $n \in \mathbb{N}$, there exist three positive constants $m_{2 n}, M_{2 n}$ and $M_{2 n+1}$ with $m_{2 n} \leq M_{2 n}$ and such that for all $u \in H$ we have

i) $m_{2 n}\|u\|_{W_{1}}^{2} \leq\left\|B_{1}^{*}(t) u\right\|_{U_{1}}^{2} \leq M_{2 n}\|u\|_{W_{1}}^{2}$ for $t \in I_{2 n}=\left[t_{2 n}, t_{2 n+1}\right), \quad \forall n \in \mathbb{N}$;

ii) $\left\|B_{2}^{*}(t) u\right\|_{U_{2}}^{2} \leq M_{2 n+1}\|u\|_{W_{2}}^{2}$ for $t \in I_{2 n+1}=\left[t_{2 n+1}, t_{2 n+2}\right), \quad \forall n \in \mathbb{N}$.

\subsection{Stability without restriction on the delay time intervals}

In this section we assume $W_{1}=W_{2}$ and we use the notation

$$
W:=W_{1}=W_{2} .
$$

Moreover, we assume

$$
\inf _{n \in \mathbb{N}} \frac{m_{2 n}}{M_{2 n+1}}>0
$$


Note that assumption (3.3) in [19] is instead equivalent to

$$
\frac{m_{2 n}}{M_{2 n+1}}>\frac{1}{2}
$$

Let us introduce the energy of the system

$$
E(t)=E(u ; t):=\frac{1}{2}\left(\|u(t)\|_{V}^{2}+\left\|u_{t}(t)\right\|_{H}^{2}+\frac{\xi}{2} \int_{t-\tau}^{t}\left\|B_{2}^{*}(s+\tau) u_{t}(s)\right\|_{U_{2}}^{2} d s\right),
$$

where $\xi$ is a positive number satisfying

$$
\xi<\inf _{n \in \mathbb{N}} \frac{m_{2 n}}{M_{2 n+1}} .
$$

Proposition 3.1 Assume i), ii) and (3.2). For any regular solution of problem (1.1) - (1.2) the energy is decreasing on the intervals $I_{2 n}, n \in \mathbb{N}$, and

$$
E^{\prime}(t) \leq-\frac{m_{2 n}}{2}\left\|u_{t}\right\|_{W}^{2}
$$

Moreover, on the intervals $I_{2 n+1}, n \in \mathbb{N}$,

$$
E^{\prime}(t) \leq \frac{M_{2 n+1}}{2}\left(\xi+\frac{1}{\xi}\right)\left\|u_{t}\right\|_{W}^{2}
$$

Proof: Differentiating $E(t)$ we get

$$
E^{\prime}(t)=\left(u_{t}, u\right)_{V}+\left(u_{t t}, u_{t}\right)_{H}+\frac{\xi}{2}\left\|B_{2}^{*}(t+\tau) u_{t}(t)\right\|_{U_{2}}^{2}-\frac{\xi}{2}\left\|B_{2}^{*}(t) u_{t}(t-\tau)\right\|_{U_{2}}^{2} .
$$

Then, using the definition of $A$ and (1.1) we obtain

$$
\begin{aligned}
E^{\prime}(t)= & \left\langle u_{t}, u_{t t}+A u\right\rangle_{V-V^{\prime}}+\frac{\xi}{2}\left\|B_{2}^{*}(t+\tau) u_{t}(t)\right\|_{U_{2}}^{2}-\frac{\xi}{2}\left\|B_{2}^{*}(t) u_{t}(t-\tau)\right\|_{U_{2}}^{2} \\
= & -\left\langle u_{t}, B_{1}(t) B_{1}^{*}(t) u_{t}(t)+B_{2}(t) B_{2}^{*}(t) u_{t}(t-\tau)\right\rangle_{V-V^{\prime}} \\
& +\frac{\xi}{2}\left\|B_{2}^{*}(t+\tau) u_{t}(t)\right\|_{U_{2}}^{2}-\frac{\xi}{2}\left\|B_{2}^{*}(t) u_{t}(t-\tau)\right\|_{U_{2}}^{2} .
\end{aligned}
$$

By the definition of the dual operators, we arrive at

$$
\begin{aligned}
E^{\prime}(t)= & -\left\|B_{1}^{*}(t) u_{t}(t)\right\|_{U_{1}}^{2}-\left(B_{2}^{*}(t) u_{t}, B_{2}^{*}(t) u_{t}(t-\tau)\right)_{U_{2}} \\
& +\frac{\xi}{2}\left\|B_{2}^{*}(t+\tau) u_{t}(t)\right\|_{U_{2}}^{2}-\frac{\xi}{2}\left\|B_{2}^{*}(t) u_{t}(t-\tau)\right\|_{U_{2}}^{2} .
\end{aligned}
$$

If $t \in I_{2 n}$, then $B_{2}(t)=0$ and the previous identity gives

$$
E^{\prime}(t)=-\left\|B_{1}^{*}(t) u_{t}(t)\right\|_{U_{1}}^{2}+\frac{\xi}{2}\left\|B_{2}^{*}(t+\tau) u_{t}(t)\right\|_{U_{2}}^{2} .
$$

Since $T_{2 n}=\left|I_{2 n}\right| \geq \tau$, it results that $t+\tau \in I_{2 n} \cup I_{2 n+1} \cup I_{2 n+2}$. Now, if $t+\tau \in I_{2 n} \cup I_{2 n+2}$, then $B_{2}(t+\tau)=0$. Therefore, $B_{2}(t+\tau) \neq 0$ only if $t+\tau \in I_{2 n+1}$. In both cases, by our assumptions i) and ii), we get (3.5). 
For $t \in I_{2 n+1}$, as $B_{1}(t)=0$, the previous identity becomes

$$
E^{\prime}(t)=-\left(B_{2}^{*}(t) u_{t}, B_{2}^{*}(t) u_{t}(t-\tau)\right)_{U_{2}}+\frac{\xi}{2}\left\|B_{2}^{*}(t+\tau) u_{t}(t)\right\|_{U_{2}}^{2}-\frac{\xi}{2}\left\|B_{2}^{*}(t) u_{t}(t-\tau)\right\|_{U_{2}}^{2} .
$$

By Young's inequality we get

$$
E^{\prime}(t) \leq \frac{1}{2 \xi}\left\|B_{2}^{*}(t) u_{t}(t)\right\|_{U_{2}}^{2}+\frac{\xi}{2}\left\|B_{2}^{*}(t+\tau) u_{t}(t)\right\|_{U_{2}}^{2}
$$

This proves (3.6) using assumption ii) because $t+\tau$ is either in $I_{2 n+1}$, or in $I_{2 n+2}$ and in that last case $B_{2}^{*}(t+\tau)=0$.

Consider now the conservative system associated with (1.1)-(1.2)

$$
\begin{aligned}
& w_{t t}(t)+A w(t)=0 \quad t>0 \\
& w(0)=w_{0} \quad \text { and } \quad w_{t}(0)=w_{1}
\end{aligned}
$$

with $\left(w_{0}, w_{1}\right) \in V \times H$. Denote by $E_{S}(\cdot)$ the standard energy for wave type equations, that is

$$
E_{S}(t)=E_{S}(w, t):=\frac{1}{2}\left(\|w\|_{V}^{2}+\left\|w_{t}\right\|_{H}^{2}\right) .
$$

For our stability result we need that a suitable observability inequality holds. Namely we assume that there exists a time $\bar{T}>0$ such that for every time $T>\bar{T}$ there is a constant $c$, depending on $T$ but independent of the initial data, such that

$$
E_{S}(0) \leq c \int_{0}^{T}\left\|w_{t}(s)\right\|_{W}^{2} d s
$$

for every weak solution of problem (3.7) $-(3.8)$ with initial data $\left(w_{0}, w_{1}\right) \in V \times H$.

Proposition 3.2 Assume i), ii) and (3.2). Moreover, we assume that the observability inequality (3.10) holds for every time $T>\bar{T}$ and that, denoting $T^{*}:=\inf _{n}\left\{T_{2 n}\right\}$, it is

$$
T^{*}>\bar{T}, \quad T^{*} \geq \tau .
$$

Then, for any solution of system (1.1) - (1.2) we have

$$
E\left(t_{2 n+1}\right) \leq c_{n} E\left(t_{2 n}\right), \quad \forall n \in \mathbb{N},
$$

where

$$
c_{n}=\frac{4 c\left(1+4 C^{2} T_{2 n}^{2} M_{2 n}^{2}\right)}{m_{2 n}+4 c\left(1+4 C^{2} T_{2 n}^{2} M_{2 n}^{2}\right)},
$$

$c$ being the observability constant in (3.10) corresponding to the time $T^{*}$ and $C$ the constant in the norm embedding (3.1) between $W$ and $H$.

Proof. It is sufficient to prove the estimate (3.12) in the interval $I_{0}=\left[0, t_{1}\right)$. We can proceed analogously in the other intervals $I_{2 n}, n \in \mathbb{N}$.

We can decompose

$$
u=w+\tilde{w}
$$


where $w$ is a solution of system (3.7) - (3.8) with $w_{0}=u_{0}, w_{1}=u_{1}$; while $\tilde{w}$ solves

$$
\begin{aligned}
& \tilde{w}_{t t}(t)+A \tilde{w}(t)=-B_{1}(t) B_{1}^{*}(t) u_{t}(t) \quad t>0 \\
& \tilde{w}(0)=0 \quad \text { and } \quad \tilde{w}_{t}(0)=0
\end{aligned}
$$

First we have

$$
E(0)=E_{S}(w, 0)+\frac{1}{2} \int_{-\tau}^{0}\left\|B_{2}^{*}(s+\tau) u_{t}(s)\right\|_{U_{2}}^{2} d s=E_{S}(w, 0),
$$

because for $s \in(-\tau, 0), s+\tau<\tau<t_{1}$. Now using the observability inequality (3.10) we can estimate

$$
E(0)=E_{S}(w, 0) \leq c \int_{0}^{T^{*}}\left\|w_{t}(s)\right\|_{W}^{2} d s .
$$

Using the splitting $w=u-\tilde{w}$ and the fact that $T_{0}=t_{1} \geq T^{*}$, we deduce that

$$
E(0) \leq 2 c \int_{0}^{T_{0}}\left(\left\|\tilde{w}_{t}(s)\right\|_{W}^{2}+\left\|u_{t}(s)\right\|_{W}^{2}\right) d s .
$$

Now, observe that from equation (3.14) we deduce

$$
\frac{d}{d t} \frac{1}{2}\left(\left\|\tilde{w}_{t}(t)\right\|_{H}^{2}+\|\tilde{w}(t)\|_{V}^{2}\right)=\left(\tilde{w}_{t}, \tilde{w}_{t t}+A \tilde{w}\right)_{H}=-\left(\tilde{w}_{t}, B_{1}(t) B_{1}^{*}(t) u_{t}(t)\right)_{H} .
$$

Integrating this identity in $[0, t]$ with $0<t<T_{0}$, recalling (3.15), and using the assumption i), we get

$$
\begin{aligned}
\frac{1}{2}\left(\left\|\tilde{w}_{t}(t)\right\|_{H}^{2}+\|\tilde{w}(t)\|_{V}^{2}\right) & =-\int_{0}^{t}\left(B_{1}^{*}(s) \tilde{w}_{t}(s), B_{1}^{*}(s) u_{t}(s)\right)_{H} d s \\
& \leq M_{0} \int_{0}^{T_{0}}\left\|\tilde{w}_{t}(s)\right\|_{W}\left\|u_{t}(s)\right\|_{W} d s .
\end{aligned}
$$

Integrating (3.18) on $\left[0, t_{1}\right]$, we deduce

$$
\begin{gathered}
\int_{0}^{T_{0}}\left\|\tilde{w}_{t}(t)\right\|_{W}^{2} d t \leq C \int_{0}^{T_{0}}\left\|\tilde{w}_{t}(t)\right\|_{H}^{2} d t \leq 2 C T_{0} M_{0} \int_{0}^{T_{0}}\left\|\tilde{w}_{t}(s)\right\|_{W}\left\|u_{t}(s)\right\|_{W} d s \\
\leq C T_{0} M_{0} \int_{0}^{T_{0}}\left(\epsilon\left\|\tilde{w}_{t}(t)\right\|_{W}^{2}+\frac{1}{\epsilon}\left\|u_{t}(t)\right\|_{W}^{2}\right) d t
\end{gathered}
$$

for all $\epsilon>0$ and therefore choosing $\epsilon$ such that $C T_{0} M_{0} \epsilon=\frac{1}{2}$, we arrive at

$$
\int_{0}^{T_{0}}\left\|\tilde{w}_{t}(t)\right\|_{W}^{2} d t \leq 4 C^{2} T_{0}^{2} M_{0}^{2} \int_{0}^{T_{0}}\left\|u_{t}(t)\right\|_{W}^{2} d t
$$

From (3.17) and (3.19) we obtain

$$
\begin{gathered}
E(0) \leq 2 c\left(1+4 C^{2} T_{0}^{2} M_{0}^{2}\right) \int_{0}^{T_{0}}\left\|u_{t}(t)\right\|_{W}^{2} d t \\
\leq \frac{4 c\left(1+4 C^{2} T_{0}^{2} M_{0}^{2}\right)}{m_{0}} \frac{m_{0}}{2} \int_{0}^{T_{0}}\left\|u_{t}(t)\right\|_{W}^{2} d t .
\end{gathered}
$$


From (3.5) and (3.20) we deduce

$$
E\left(t_{1}\right) \leq E(0) \leq \frac{4 c\left(1+4 C^{2} T_{0}^{2} M_{0}^{2}\right)}{m_{0}}\left(E(0)-E\left(t_{1}\right)\right)
$$

where we used also the fact that $E(\cdot)$ is decreasing on the time interval $\left[0, t_{1}\right]$. This clearly implies

$$
E\left(t_{1}\right) \leq c_{0} E(0)
$$

with

$$
c_{0}=\frac{4 c\left(1+4 C^{2} T_{0}^{2} M_{0}^{2}\right)}{m_{0}+4 c\left(1+4 C^{2} T_{0}^{2} M_{0}^{2}\right)} .
$$

Theorem 3.3 Under the assumptions of Proposition 3.2. if

$$
\sum_{n=0}^{\infty} M_{2 n+1} T_{2 n+1}<+\infty \quad \text { and } \quad \sum_{n=0}^{\infty} \frac{m_{2 n}}{1+4 C^{2} T_{2 n}^{2} M_{2 n}^{2}}=+\infty,
$$

then system (5.3) - (5.6) is asymptotically stable, that is any solution $u$ of (5.3) - (5.6) satisfies $E(u, t) \rightarrow 0$ for $t \rightarrow+\infty$.

Proof. Note that (3.6) implies

$$
E^{\prime}(t) \leq M_{2 n+1}\left(\xi+\frac{1}{\xi}\right) C E(t), \quad t \in I_{2 n+1}=\left[t_{2 n+1}, t_{2 n+2}\right), n \in \mathbb{N} .
$$

Then we have

$$
E\left(t_{2 n+2}\right) \leq e^{C\left(\xi+\frac{1}{\xi}\right) M_{2 n+1} T_{2 n+1}} E\left(t_{2 n+1}\right), \quad \forall n \in \mathbb{N} .
$$

Combining Proposition 3.2 and (3.22) we obtain

$$
E\left(t_{2 n+2}\right) \leq e^{C\left(\xi+\frac{1}{\xi}\right) M_{2 n+1} T_{2 n+1}} c_{n} E\left(t_{2 n}\right), \quad n \in \mathbb{N},
$$

and therefore

$$
E\left(t_{2 n+2}\right) \leq\left(\Pi_{p=0}^{n} e^{C\left(\xi+\frac{1}{\xi}\right) M_{2 p+1} T_{2 p+1}} c_{p}\right) E(0) .
$$

Then, by (3.23), asymptotic stability occurs if

$$
\sum_{p=0}^{\infty}\left[C\left(\xi+\frac{1}{\xi}\right) M_{2 p+1} T_{2 p+1}+\ln c_{p}\right]=-\infty .
$$

In particular (3.24) holds true if (3.21) is valid. Indeed, from (3.13),

$$
c_{p}=\frac{1}{\frac{m_{2 p}}{4 c\left(1+4 C^{2} T_{2 p}^{2} M_{2 p}^{2}\right)}+1},
$$

and then

$$
\ln c_{p}=-\ln \left(1+\frac{m_{2 p}}{4 c\left(1+4 C^{2} T_{2 p}^{2} M_{2 p}^{2}\right)}\right) .
$$


So, if $\frac{m_{2 p}}{1+4 C^{2} T_{2 p}^{2} M_{2 p}^{2}}$ tends to 0 as $p \rightarrow \infty$, then

$$
-\ln c_{p} \sim \frac{m_{2 p}}{4 c\left(1+4 C^{2} T_{2 p}^{2} M_{2 p}^{2}\right)}
$$

Consequently if (3.21) holds then

$$
\sum_{p=0}^{\infty} \ln c_{p}=-\infty .
$$

Otherwise, if $\frac{m_{2 p}}{1+4 C^{2} T_{2 p}^{2} M_{2 p}^{2}}$ does not tend to 0 , then, by (3.25), $\sum_{p=0}^{\infty} \ln c_{p}=-\infty$. Therefore, conditions (3.21) imply (3.24).

We now show that under additional assumptions on the coefficients $T_{n}, m_{n}, M_{n}$ an exponential stability result holds.

Theorem 3.4 Assume i), ii) and (3.2). Assume also that the observability inequality (3.10) holds for every time $T>\bar{T}$ and that

$$
T_{2 n}=T^{*} \quad \forall n \in \mathbb{N},
$$

with $T^{*}$ satisfying (3.11), and

$$
T_{2 n+1}=\tilde{T} \quad \forall n \in \mathbb{N} .
$$

Moreover, assume that

$$
\sup _{n \in \mathbb{N}} e^{\left(\xi+\frac{1}{\xi}\right) C M_{2 n+1} \tilde{T}} c_{n}=d<1,
$$

where $c_{n}$ is as in (3.13). Then, there exist two positive constants $\gamma, \mu$ such that

$$
E(t) \leq \gamma e^{-\mu t} E(0), \quad t>0,
$$

for any solution of problem (1.1) - (1.2).

Proof. From (3.28) and (3.23) we obtain

$$
E\left(T^{*}+\tilde{T}\right) \leq d E(0)
$$

and also

$$
E\left(n\left(T^{*}+\tilde{T}\right)\right) \leq d^{n} E(0), \quad \forall n \in \mathbb{N} .
$$

Then, the energy satisfies an exponential estimate like (3.29) (see Lemma 1 of [6]).

Remark 3.5 In the assumptions of Theorem [3.4, from (3.23) we can see that exponential stability also holds if instead of (3.28) we assume

$$
\exists n \in \mathbb{N} \quad \text { such that } \quad \Pi_{p=k(n+1)}^{k(n+1)+n} e^{\left(\xi+\frac{1}{\xi}\right) C M_{2 p+1} \tilde{T}} c_{p} \leq d<1, \quad \forall k=0,1,2, \ldots
$$

Remark 3.6 Our abstract results can be applied to the examples of [19], that is damped or locally damped wave equations, elasticity system, Petrovsky system. Therefore, we can improve the stability results for these models. 


\subsection{Stability under the restriction $T_{2 n+1} \leq \tau$}

We assume now that the length of the delay intervals is lower that the time delay, that is

$$
T_{2 n+1} \leq \tau, \quad \forall n \in \mathbb{N} .
$$

We look at the standard energy $E_{S}(\cdot)$. We can give the following estimates on the time intervals $I_{2 n}, I_{2 n+1}, n \in \mathbb{N}$.

Proposition 3.7 Assume i), ii) and (3.30). For any regular solution of problem (1.1) - (1.2) the energy is decreasing on the intervals $I_{2 n}, n \in \mathbb{N}$, and

$$
E_{S}^{\prime}(t) \leq-m_{2 n}\left\|u_{t}(t)\right\|_{W_{1}}^{2} .
$$

Moreover, on the intervals $I_{2 n+1}, n \in \mathbb{N}$,

$$
E_{S}^{\prime}(t) \leq \frac{M_{2 n+1}}{2}\left\|u_{t}(t)\right\|_{W_{2}}^{2}+\frac{M_{2 n+1}}{2}\left\|u_{t}(t-\tau)\right\|_{W_{2}}^{2} .
$$

Proof: Differentiating $E_{S}(t)$ we get

$$
E_{S}^{\prime}(t)=\left(u_{t}, u\right)_{V}+\left(u_{t t}, u_{t}\right)_{H}
$$

Hence using the definition of $A$ and (1.1) we get successively

$$
\begin{aligned}
E_{S}^{\prime}(t) & =\left\langle u_{t}, u_{t t}+A u\right\rangle_{V-V^{\prime}} \\
& =-\left\langle u_{t}, B_{1}(t) B_{1}^{*}(t) u_{t}(t)+B_{2}(t) B_{2}^{*}(t) u_{t}(t-\tau)\right\rangle_{V-V^{\prime}} .
\end{aligned}
$$

By the definition of the dual operators, we arrive at

$$
E_{S}^{\prime}(t)=-\left\|B_{1}^{*}(t) u_{t}(t)\right\|_{U_{1}}^{2}-\left(B_{2}^{*}(t) u_{t}, B_{2}^{*}(t) u_{t}(t-\tau)\right)_{U_{2}} .
$$

If $t \in I_{2 n}$, then $B_{2}(t)=0$ and the previous identity becomes

$$
E_{S}^{\prime}(t)=-\left\|B_{1}^{*}(t) u_{t}(t)\right\|_{U_{1}}^{2} .
$$

This gives, from i), (3.31).

For $t \in I_{2 n+1}$, as $B_{1}(t)=0$, the previous identity gives

$$
E_{S}^{\prime}(t)=-\left(B_{2}^{*}(t) u_{t}, B_{2}^{*}(t) u_{t}(t-\tau)\right)_{U_{2}} \leq \frac{1}{2}\left\|B_{2}^{*}(t) u_{t}(t)\right\|_{U_{2}}^{2}+\frac{1}{2}\left\|B_{2}^{*}(t) u_{t}(t-\tau)\right\|_{U_{2}}^{2} .
$$

This proves (3.32) using assumption ii).

Proposition 3.8 Assume i), ii) and (3.30). Moreover, we assume that the observability inequality (3.10) holds for every time $T>\bar{T}$ and that, denoting $T^{*}:=\inf _{n}\left\{T_{2 n}\right\}$, (3.11) is satisfied. Then, for any solution of system (1.1) - (1.2) we have

$$
E_{S}\left(t_{2 n+1}\right) \leq \hat{c}_{n} E_{S}\left(t_{2 n}\right), \quad \forall n \in \mathbb{N},
$$

where

$$
\hat{c}_{n}=\frac{2 c\left(1+4 C_{1}^{2} T_{2 n}^{2} M_{2 n}^{2}\right)}{m_{2 n}+2 c\left(1+4 C_{1}^{2} T_{2 n}^{2} M_{2 n}^{2}\right)},
$$

$c$ being the observability constant in (3.10) corresponding to the time $T^{*}$ and $C_{1}$ the constant in the norm embedding (3.1) between $W_{1}$ and $H$. 
Proof. It is sufficient to prove the estimate (3.33) in the interval $I_{0}=\left[0, t_{1}\right)$. We can proceed analogously in the other intervals $I_{2 n}, n \in \mathbb{N}$.

We can decompose

$$
u=w+\tilde{w}
$$

where $w$ is a solution of system (3.7) - (3.8) with $w_{0}=u_{0}, w_{1}=u_{1}$; while $\tilde{w}$ solves

$$
\begin{aligned}
& \tilde{w}_{t t}(t)+A \tilde{w}(t)=-B_{1}(t) B_{1}^{*}(t) u_{t}(t) \quad t>0 \\
& \tilde{w}(0)=0 \quad \text { and } \quad \tilde{w}_{t}(0)=0
\end{aligned}
$$

First we have

$$
E_{S}(u, 0)=E_{S}(w, 0) .
$$

Then, using the observability inequality (3.10), we can estimate

$$
E_{S}(0)=E_{S}(w, 0) \leq c \int_{0}^{T^{*}}\left\|w_{t}(s)\right\|_{W_{1}}^{2} d s .
$$

Using the splitting $w=u-\tilde{w}$ and the fact that $T_{0}=t_{1} \geq T^{*}$, we deduce that

$$
E_{S}(0) \leq 2 c \int_{0}^{T_{0}}\left(\left\|\tilde{w}_{t}(s)\right\|_{W_{1}}^{2}+\left\|u_{t}(s)\right\|_{W_{1}}^{2}\right) d s .
$$

Now, observe that from equation (3.14) we deduce

$$
\frac{d}{d t} \frac{1}{2}\left(\left\|\tilde{w}_{t}(t)\right\|_{H}^{2}+\|\tilde{w}(t)\|_{V}^{2}\right)=\left(\tilde{w}_{t}, \tilde{w}_{t t}+A \tilde{w}\right)_{H}=-\left(\tilde{w}_{t}, B_{1}(t) B_{1}^{*}(t) u_{t}(t)\right)_{H} .
$$

Integrating this identity in $[0, t]$ with $0<t<T_{0}$, recalling (3.15), and using the assumption i), we get

$$
\begin{aligned}
\frac{1}{2}\left(\left\|\tilde{w}_{t}(t)\right\|_{H}^{2}+\|\tilde{w}(t)\|_{V}^{2}\right) & =-\int_{0}^{t}\left(B_{1}^{*}(s) \tilde{w}_{t}(s), B_{1}^{*}(s) u_{t}(s)\right)_{H} d s \\
& \leq M_{0} \int_{0}^{T_{0}}\left\|\tilde{w}_{t}(s)\right\|_{W_{1}}\left\|u_{t}(s)\right\|_{W_{1}} d s .
\end{aligned}
$$

Integrating (3.39) on $\left[0, t_{1}\right]$, we deduce

$$
\begin{gathered}
\int_{0}^{T_{0}}\left\|\tilde{w}_{t}(t)\right\|_{W_{1}}^{2} d t \leq C_{1} \int_{0}^{T_{0}}\left\|\tilde{w}_{t}(t)\right\|_{H}^{2} d t \leq 2 C_{1} T_{0} M_{0} \int_{0}^{T_{0}}\left\|\tilde{w}_{t}(s)\right\|_{W_{1}}\left\|u_{t}(s)\right\|_{W_{1}} d s \\
\leq C_{1} T_{0} M_{0} \int_{0}^{T_{0}}\left(\epsilon\left\|\tilde{w}_{t}(t)\right\|_{W_{1}}^{2}+\frac{1}{\epsilon}\left\|u_{t}(t)\right\|_{W_{1}}^{2}\right) d t
\end{gathered}
$$

for all $\epsilon>0$ and therefore choosing $\epsilon$ such that $C_{1} T_{0} M_{0} \epsilon=\frac{1}{2}$, we arrive at

$$
\int_{0}^{T_{0}}\left\|\tilde{w}_{t}(t)\right\|_{W_{1}}^{2} d t \leq 4 C_{1}^{2} T_{0}^{2} M_{0}^{2} \int_{0}^{T_{0}}\left\|u_{t}(t)\right\|_{W_{1}}^{2} d t .
$$

From (3.38) and (3.40) we obtain

$$
\begin{gathered}
E_{S}(0) \leq 2 c\left(1+4 C_{1}^{2} T_{0}^{2} M_{0}^{2}\right) \int_{0}^{T_{0}}\left\|u_{t}(t)\right\|_{W_{1}}^{2} d t \\
\quad \leq \frac{2 c\left(1+4 C_{1}^{2} T_{0}^{2} M_{0}^{2}\right)}{m_{0}} m_{0} \int_{0}^{T_{0}}\left\|u_{t}(t)\right\|_{W_{1}}^{2} d t .
\end{gathered}
$$


From (3.31) and (3.41) we deduce

$$
E_{S}\left(t_{1}\right) \leq E_{S}(0) \leq \frac{2 c\left(1+4 C_{1}^{2} T_{0}^{2} M_{0}^{2}\right)}{m_{0}}\left(E_{S}(0)-E_{S}\left(t_{1}\right)\right)
$$

where we used also the fact that $E_{S}(\cdot)$ is decreasing on the time interval $\left[0, t_{1}\right]$. This clearly implies

$$
E_{S}\left(t_{1}\right) \leq \hat{c}_{0} E_{S}(0)
$$

with

$$
\hat{c}_{0}=\frac{2 c\left(1+4 C_{1}^{2} T_{0}^{2} M_{0}^{2}\right)}{m_{0}+2 c\left(1+4 C_{1}^{2} T_{0}^{2} M_{0}^{2}\right)} .
$$

Theorem 3.9 Under the assumptions of Proposition 3.8, if (3.21) holds, then system (1.1) (1.2) is asymptotically stable, that is any solution $u$ of (1.1) - (1.2) satisfies $E_{S}(u, t) \rightarrow 0$ for $t \rightarrow+\infty$.

Proof. Note that (3.32) implies

$$
\begin{aligned}
E_{S}^{\prime}(t) & \leq M_{2 n+1} C_{2} E_{S}(t)+M_{2 n+1} C_{2} E_{S}(t-\tau) \\
& \leq M_{2 n+1} C_{2} E_{S}(t)+M_{2 n+1} C_{2} E_{S}\left(t_{2 n+1}\right), \quad t \in I_{2 n+1}=\left[t_{2 n+1}, t_{2 n+2}\right), n \in \mathbb{N},
\end{aligned}
$$

where we have used (3.30) and the fact that $E_{S}(\cdot)$ is not increasing in the time intervals $I_{2 n}$. Remark that the constant $C_{2}$ is the one from the norm embedding (3.1) between $W_{2}$ and $H$.

Then we have

$$
E_{S}(t) \leq e^{M_{2 n+1} C_{2}\left(t-t_{2 n+1}\right)} E_{S}\left(t_{2 n+1}\right)+\left[e^{M_{2 n+1} C_{2}\left(t-t_{2 n+1}\right)}-1\right] E_{S}\left(t_{2 n}\right),
$$

for $t \in I_{2 n+1}=\left[t_{2 n+1}, t_{2 n+2}\right), n \in \mathbb{N}$. Combining Proposition 3.8 and (3.42) we obtain

$$
E_{S}\left(t_{2 n+2}\right) \leq\left[e^{M_{2 n+1} T_{2 n+1} C_{2}} \hat{c}_{n}+e^{M_{2 n+1} T_{2 n+1} C_{2}}-1\right] E_{S}\left(t_{2 n}\right), \quad n \in \mathbb{N},
$$

and therefore

$$
E_{S}\left(t_{2 n+2}\right) \leq\left(\Pi_{p=0}^{n}\left[e^{M_{2 p+1} T_{2 p+1} C_{2}} \hat{c}_{p}+e^{M_{2 p+1} T_{2 p+1} C_{2}}-1\right]\right) E_{S}(0) .
$$

Then, by (3.43), asymptotic stability occurs if

$$
\sum_{p=0}^{\infty}\left[C_{2} M_{2 p+1} T_{2 p+1}+\ln \left(\hat{c}_{p}+1-e^{-M_{2 p+1} C_{2} T_{2 p+1}}\right)\right]=-\infty .
$$

In particular (3.44) holds true if (3.21) is valid. Indeed, if (3.21) holds, then

$$
\lim _{n \rightarrow \infty} M_{2 n+1} T_{2 n+1}=0,
$$

and therefore, being from (3.34),

$$
\hat{c}_{p}=\frac{1}{\frac{m_{2 p}}{2 c\left(1+4 C_{1}^{2} T_{2 p}^{2} M_{2 p}^{2}\right)}+1},
$$


it results

$$
\ln \left(\hat{c}_{p}+1-e^{-M_{2 p+1} C_{2} T_{2 p+1}}\right) \sim \ln \hat{c}_{p}=-\ln \left(1+\frac{m_{2 p}}{2 c\left(1+4 C_{1}^{2} T_{2 p}^{2} M_{2 p}^{2}\right)}\right) .
$$

So, if $\frac{m_{2 p}}{1+4 C_{1}^{2} T_{2 p}^{2} M_{2 p}^{2}}$ tends to 0 as $p \rightarrow \infty$, then

$$
-\ln \hat{c}_{p} \sim \frac{m_{2 p}}{2 c\left(1+4 C_{1}^{2} T_{2 p}^{2} M_{2 p}^{2}\right)}
$$

Otherwise, if $\frac{m_{2 p}}{1+4 C_{1}^{2} T_{2 p}^{2} M_{2 p}^{2}}$ does not tend to 0 , then, by (3.45), $\sum_{p=0}^{\infty} \ln \hat{c}_{p}=-\infty$. Therefore, conditions (3.21) imply

$$
\sum_{p=0}^{\infty} \ln \hat{c}_{p}=-\infty
$$

and then (3.44).

Also in this case, under additional assumptions on the coefficients $T_{n}, m_{n}, M_{n}$, an exponential stability result holds.

Theorem 3.10 Assume i), ii) and (3.30). Assume also that the observability inequality (3.10) holds for every time $T>\bar{T}$ and that

$$
T_{2 n}=T^{*} \quad \forall n \in \mathbb{N},
$$

with $T^{*}$ satisfying (3.11), and

$$
T_{2 n+1}=\tilde{T} \quad \forall n \in \mathbb{N},
$$

with $\tilde{T} \leq \tau$.

Moreover, assume that

$$
\sup _{n \in \mathbb{N}}\left[e^{C_{2} M_{2 n+1} \tilde{T}}\left(\hat{c}_{n}+1\right)-1\right]=\hat{d}<1
$$

where $\hat{c}_{n}$ is as in (3.34). Then, there exist two positive constants $\hat{\gamma}, \hat{\mu}$ such that

$$
E_{S}(t) \leq \hat{\gamma} e^{-\hat{\mu} t} E_{S}(0), \quad t>0,
$$

for any solution of problem (1.1) - (1.2).

Remark 3.11 In the assumptions of Theorem 3.10, from (3.43) we can see that exponential stability also holds if instead of (3.48) we assume

$$
\exists n \in \mathbb{N} \quad \text { such that } \quad \Pi_{p=k(n+1)}^{k(n+1)+n}\left[e^{C_{2} M_{2 p+1} \tilde{T}}\left(\hat{c}_{p}+1\right)-1\right] \leq \hat{d}<1, \quad \forall k=0,1,2, \ldots
$$

Remark 3.12 Our abstract results can be applied to the examples of [19], that is damped or locally damped wave equations, elasticity system, Petrovsky system when $T_{2 n+1} \leq \tau, \forall n \in \mathbb{N}$. 


\section{Stability result: $B_{1}$ unbounded}

In this section $B_{1}$ may be unbounded. We assume that there exists a Hilbert space $W$ such that $H$ is continuously embedded into $W$, i.e.,

$$
\|u\|_{W}^{2} \leq C\|u\|_{H}^{2}, \quad \forall u \in H \text { with } C>0 \text { independent of } u .
$$

Moreover, we assume that $V$ is embedded into $U_{1}$ and that for all $n \in \mathbb{N}$, there exist three positive constants $m_{2 n}, M_{2 n}$ and $M_{2 n+1}$ with $m_{2 n} \leq M_{2 n}$ such that

i) $m_{2 n}\|u\|_{U_{1}}^{2} \leq\left\|B_{1}^{*}(t) u\right\|_{U_{1}}^{2} \leq M_{2 n}\|u\|_{U_{1}}^{2}$ for $t \in I_{2 n}=\left[t_{2 n}, t_{2 n+1}\right), \quad \forall u \in V, \quad \forall n \in \mathbb{N}$;

ii) $\left\|B_{2}^{*}(t) u\right\|_{U_{2}}^{2} \leq M_{2 n+1}\|u\|_{W}^{2}$ for $t \in I_{2 n+1}=\left[t_{2 n+1}, t_{2 n+2}\right), \quad \forall u \in H, \quad \forall n \in \mathbb{N}$.

In order to deal with unbounded feedback we will work with the standard energy $E_{S}(\cdot)$. Then, as before, we assume (3.30).

As before we can give the following estimates on the time intervals $I_{2 n}, I_{2 n+1}, n \in \mathbb{N}$.

Proposition 4.1 Assume i), ii) and (3.30). For any regular solution of problem (1.1) - (1.2) the energy is decreasing on the intervals $I_{2 n}, n \in \mathbb{N}$, and

$$
E_{S}^{\prime}(t) \leq-\left\|B_{1}^{*}(t) u_{t}(t)\right\|_{U_{1}}^{2} .
$$

Moreover, on the intervals $I_{2 n+1}, n \in \mathbb{N}$, the estimate (3.32) holds (with $W$ instead of $W_{2}$.)

Consider now the damped system

$$
\begin{aligned}
& w_{t t}(t)+A w(t)+B_{1}(t) B_{1}^{*}(t) w_{t}=0, \quad t \in\left(t_{2 n}, t_{2 n+1}\right), n \in \mathbb{N}, \\
& w\left(t_{2 n}\right)=w_{0}^{n} \quad \text { and } \quad w_{t}\left(t_{2 n}\right)=w_{1}^{n}
\end{aligned}
$$

with $\left(w_{0}^{n}, w_{1}^{n}\right) \in V \times H$. For our stability result we need that the next observability type inequality holds. Namely we assume that, for every $n$ there exists a time $\bar{T}_{n}$, such that

$$
T_{2 n}>\bar{T}_{n},
$$

and for every $n$ and every time $T$, with $T_{2 n} \geq T>\bar{T}_{n}$, there is a constant $d_{n}$, depending on $T$ but independent of $\left(w_{0}^{n}, w_{1}^{n}\right)$, such that

$$
E_{S}\left(t_{2 n}+T\right) \leq d_{n} \int_{t_{2 n}}^{t_{2 n}+T}\left\|B_{1}^{*}(t) w_{t}(t)\right\|_{U_{1}}^{2} d t,
$$

for every weak solution of problem (1.1), (1.2) with initial data $\left(w_{0}^{n}, w_{1}^{n}\right) \in V \times H$.

Proposition 4.2 Assume i), ii), (3.30) and $T_{2 n} \geq \tau, \forall n \in \mathbb{N}$. Moreover, we assume that there is a sequence $\left\{\bar{T}_{n}\right\}_{n}$, such that (4.5) is satisfied and the inequality (4.6) holds for every $T \in$ $\left(\bar{T}_{n}, T_{2 n}\right], \forall n \in \mathbb{N}$. Then, for any solution of system (1.1) - (1.2) we have

$$
E_{S}\left(t_{2 n+1}\right) \leq \hat{d}_{n} E_{S}\left(t_{2 n}\right), \quad \forall n \in \mathbb{N},
$$

where

$$
\hat{d}_{n}=\frac{d_{n}}{d_{n}+1},
$$

$d_{n}$ being the observability constant in (4.6) corresponding to the time $T_{2 n}$. 
Proof. To prove (4.7) is sufficient to use the estimate (4.2) in (4.6), reminding that $B_{2}(t)=0$ on $\left(t_{2 n}, t_{2 n+1}\right)$.

Theorem 4.3 Under the assumptions and with the same notations of Proposition 4.2, if

$$
\sum_{n=0}^{\infty} M_{2 n+1} T_{2 n+1}<+\infty \text { and } \sum_{n=0}^{\infty} \ln \hat{d}_{n}=-\infty,
$$

then system (1.1) - (1.2) is asymptotically stable, that is any solution $u$ of (1.1) - (1.2) satisfies $E_{S}(u, t) \rightarrow 0$ for $t \rightarrow+\infty$.

Remark 4.4 In fact $d_{n}$ depends on $n$ because by hypothesis $B_{1}$ may depend on the time variable. However, if $B_{1}$ does not depend on $t$, then by a translation of $t_{2 n}$ the constant $d_{n}$ becomes independent of $n$. But if $d_{n}=d>0$ for all $n$, then the condition

$$
\sum_{n=0}^{\infty} \ln \hat{d}_{n}=-\infty
$$

is automatically satisfied. On the other hand, the first condition in (4.9) depends only on the length of the intervals $I_{2 n+1}$ and on the boundedness constant of $B_{2}^{*}$ on the same intervals, hence (4.9) can be easily checked.

Also in this case, under additional assumptions on the coefficients $T_{n}, m_{n}, M_{n}$, an exponential stability result holds.

Theorem 4.5 Assume i), ii) and (3.30). Assume also that (3.46) holds with $T^{*}$ satisfying $T^{*} \geq \tau$ and that inequality (4.6) holds, $\forall n \in \mathbb{N}$, for every time $T$ with $T^{*} \geq T>\bar{T}$. Moreover, assume $T_{2 n+1}=\tilde{T}$, for all $n \in \mathbb{N}$, with $\tilde{T} \leq \tau$. If

$$
\sup _{n \in \mathbb{N}}\left[e^{C M_{2 n+1} \tilde{T}}\left(\hat{d}_{n}+1\right)-1\right]<1,
$$

where $\hat{d}_{n}$ is as in (4.8), then, there exist two positive constants $\hat{\gamma}, \hat{\mu}$ such that

$$
E_{S}(t) \leq \hat{\gamma} e^{-\hat{\mu} t} E_{S}(0), \quad t>0,
$$

for any solution of problem (1.1) - (1.2).

Remark 4.6 If $\hat{d}_{n} \leq \hat{d}<1$ (see Remark 4.4), then (4.10) holds if

$$
(1+\hat{d}) \sup _{n \in \mathbb{N}}\left[e^{C M_{2 n+1} \tilde{T}}-\frac{1}{1+\hat{d}}\right]<1,
$$

or equivalently

$$
\sup _{n \in \mathbb{N}}\left[e^{C M_{2 n+1} \tilde{T}}\right]<\frac{2}{\hat{d}+1}
$$

Hence (4.10) is verified if $\sup _{n \in \mathbb{N}} M_{2 n+1}$ is small enough. This is a quite realistic assumption because then the influence of the delay term is small and the action of the standard dissipation sufficiently compensates it to guarantee an exponential decay. 


\section{$5 \quad$ Examples}

Here we apply our abstract results to some concrete models. Note that the following examples are not included in the setting of [19].

\subsection{The wave equation with internal and boundary dampings}

Our first application concerns the wave equation with boundary feedback and internal delay term. Let $\Omega \subset \mathbb{R}^{n}$ be an open bounded domain with a boundary $\partial \Omega$ of class $C^{2}$. We assume that $\partial \Omega$ is composed of two closed sets $\partial \Omega=\Gamma_{0} \cup \Gamma_{1}$, with $\Gamma_{0} \cap \Gamma_{1}=\emptyset$ and meas $\Gamma_{1}>0$.

Denoting by $m$ the standard multiplier $m(x)=x-x_{0}, x_{0} \in \mathbb{R}^{n}$, we assume that the

$$
m(x) \cdot \nu(x) \leq 0, \quad \text { for } x \in \Gamma_{1},
$$

and, for some $\delta>0$,

$$
m(x) \cdot \nu(x) \geq \delta, \quad \text { for } x \in \Gamma_{0},
$$

where $\nu(x)$ is the outer unit normal vector at $x \in \partial \Omega$. Given $\omega \subseteq \Omega$, let us consider the initial boundary value problem

$$
\begin{aligned}
& u_{t t}(x, t)-\Delta u(x, t)+b_{2}(t) \chi_{\omega} u_{t}(x, t-\tau)=0 \text { in } \Omega \times(0,+\infty) \\
& u(x, t)=0 \text { on } \Gamma_{1} \times(0,+\infty) \\
& \frac{\partial u}{\partial \nu}(x, t)=-b_{1}(t) u_{t}(x, t) \text { on } \Gamma_{0} \times(0,+\infty) \\
& u(x, 0)=u_{0}(x) \text { and } u_{t}(x, 0)=u_{1}(x) \text { in } \Omega
\end{aligned}
$$

with initial data $\left(u_{0}, u_{1}\right) \in H_{\Gamma_{1}}^{1}(\Omega) \times L^{2}(\Omega)$, where as usual

$$
H_{\Gamma_{1}}^{1}(\Omega):=\left\{u \in H^{1}(\Omega): u=0 \text { on } \Gamma_{1}\right\},
$$

and $b_{1}, b_{2}$ in $L^{\infty}(0,+\infty)$.

On the feedback functions $b_{1}(\cdot), b_{2}(\cdot)$, we assume

$$
b_{1}(t) b_{2}(t)=0, \quad \forall t>0,
$$

in order to have an intermittent delay problem. We refer to [1] for the analysis of this problem when $b_{1}, b_{2}$ are constant in time, in other words the delayed damping and the standard boundary one are acting simultaneously for every time $t>0$.

Moreover, we assume $b_{1} \in W^{2, \infty}\left(I_{2 n}\right), \forall n \in \mathbb{N}$, and

$\left.i_{w}\right) 0<m_{2 n} \leq b_{1}(t) \leq M_{2 n}, b_{2}(t)=0$, for all $t \in I_{2 n}=\left[t_{2 n}, t_{2 n+1}\right)$, and $b_{1} \in C^{1}\left(\bar{I}_{2 n}\right)$, for all $n \in \mathbb{N}$; $n \in \mathbb{N}$.

$\left.i i_{w}\right)\left|b_{2}(t)\right| \leq M_{2 n+1}, b_{1}(t)=0$, for all $t \in I_{2 n+1}=\left[t_{2 n+1}, t_{2 n+2}\right)$, and $b_{2} \in C\left(\bar{I}_{2 n+1}\right)$, for all

This problem enters into our previous framework, if we take $H=L^{2}(\Omega)$ and the operator $A$ defined by

$$
A: \mathcal{D}(A) \rightarrow H: u \rightarrow-\Delta u
$$

where

$$
\mathcal{D}(A)=:\left\{u \in H_{\Gamma_{1}}^{1}(\Omega): \Delta u \in L^{2}(\Omega) \text { and } \frac{\partial u}{\partial \nu}=0 \text { on } \Gamma_{0}\right\}
$$


We then define $U_{1}:=L^{2}\left(\Gamma_{0}\right), U_{2}:=L^{2}(\omega)$ and the operators $B_{1}(t), B_{2}(t)$ as

$$
B_{2} \in \mathcal{L}\left(U_{2} ; H\right), B_{2} u=\sqrt{b_{2}} \tilde{u}, \forall u \in L^{2}(\omega),
$$

and

$$
B_{1} \in \mathcal{L}\left(U_{1} ; V^{\prime}\right), B_{1} u=\sqrt{b_{1}} A_{-1} N u, \forall u \in L^{2}\left(\Gamma_{0}\right), B_{1}^{*} w=\sqrt{b_{1}} w_{\mid \Gamma_{0}}, \forall w \in V:=\mathcal{D}\left(A^{1 / 2}\right),
$$

where $A_{-1}$ is the extension of $A$ to $H$, namely for all $h \in H$ and $\varphi \in \mathcal{D}(A), A_{-1} h$ is the unique element in $(\mathcal{D}(A))^{\prime}$ (the duality is in the sense of $H$ ), such that (see for instance [21])

$$
\left\langle A_{-1} h ; \varphi\right\rangle_{(\mathcal{D}(A))^{\prime}, \mathcal{D}(A)}=\int_{\Omega} h A \varphi d x .
$$

Here and below $N \in \mathcal{L}\left(L^{2}\left(\Gamma_{0}\right) ; L^{2}(\Omega)\right)$ is defined as follows: for all $v \in L^{2}\left(\Gamma_{0}\right), N v$ is the unique solution (transposition solution) of

$$
\Delta N v=0, N v_{\mid \Gamma_{1}}=0, \frac{\partial N v}{\partial \nu}_{\mid \Gamma_{0}}=v
$$

With these definitions, we can show that problem (5.3)-(5.6) enters in the abstract framework (1.1) -(1.2) and that the assumptions i) and ii) of section 4 hold with $W=L^{2}(\omega)$.

As $B_{1}$ is not bounded, we need to consider the non delayed system

$$
\begin{aligned}
& w_{t t}(x, t)-\Delta w(x, t)=0 \quad \text { in } \quad \Omega \times(0,+\infty) \\
& w(x, t)=0 \quad \text { on } \quad \Gamma_{1} \times(0,+\infty) \\
& \frac{\partial w}{\partial \nu}(x, t)=-f(t) w_{t}(x, t), \quad x \in \Gamma_{0}, t>0 \\
& w(x, 0)=w_{0}(x) \text { and } w_{t}(x, 0)=w_{1}(x) \quad \text { in } \quad \Omega
\end{aligned}
$$

with $\left(w_{0}, w_{1}\right) \in H_{\Gamma_{1}}^{1}(\Omega) \times L^{2}(\Omega)$ and $f \in L^{\infty}(0,+\infty), f(t) \geq 0$ a.e. $t>0$.

Proposition 5.1 There exists a time $\bar{T}>0$ such that for every $T>\bar{T}$, there are constants $\alpha_{i}, i=1,2,3$, for which

$(T-\bar{T}) E_{S}(T) \leq \alpha_{1} \int_{0}^{T} \int_{\Gamma_{0}}\left(\frac{\partial w}{\partial \nu}\right)^{2}(x, t) d \Gamma d t+\alpha_{2} \int_{0}^{T} \int_{\Gamma_{0}} f(t) w_{t}^{2}(x, t) d \Gamma d t+\alpha_{3} \int_{0}^{T} \int_{\Gamma_{0}} w_{t}^{2}(x, t) d \Gamma d t$

for any weak solution of (5.7) - (5.10). The constants $\alpha_{i}, i=1,2,3$, are independent of the initial data and of the function $f(\cdot)$, but they depend on $T$ and on $\Omega$.

Proof. The estimate (5.11) can be easily obtained from a standard multiplier argument (cfr. (3.11)-(3.16) of [12]).

Then, from Proposition 5.1, we deduce that there exists a time $\bar{T}>0$ such that, assuming $T_{2 n}>\bar{T}, \forall n \in \mathbb{N}$, then for every $n$ and every time $T$ with $T_{2 n} \geq T>\bar{T}$, there is a constant $d_{n}$ for which (4.6) holds for any weak solution of (5.7) - (5.10).

From (5.11) with $f(t)=b_{1}\left(t-t_{2 n}\right)$ and the boundary condition (5.5) we deduce the explicit dependence of $d_{n}$ from the feedback function $b_{1}$, that is 


$$
E_{S}\left(t_{2 n+1}\right) \leq d_{n} \int_{t_{2 n}}^{t_{2 n+1}} \int_{\Gamma_{0}} b_{1}(t) u_{t}^{2}(x, t) d t d \Gamma, \forall n \in \mathbb{N}
$$

with

$$
d_{n}:=\frac{\alpha_{1} M_{2 n} m_{2 n}+\alpha_{2} m_{2 n}+\alpha_{3}}{m_{2 n}\left(T_{2 n}-\bar{T}\right)}, \quad \forall n \in \mathbb{N} .
$$

Therefore, we can restate Theorem 4.3 under more explicit conditions.

Theorem 5.2 Under the assumptions $\left.\left.i_{w}\right), i i_{w}\right)$ and (3.30), if $T^{*}:=\inf _{n} T_{2 n}$ satisfies (3.11) and

$$
\sum_{n=0}^{\infty} M_{2 n+1} T_{2 n+1}<+\infty \quad \text { and } \quad \sum_{n=0}^{\infty} \frac{m_{2 n}\left(T_{2 n}-\bar{T}\right)}{\alpha_{1} M_{2 n} m_{2 n}+\alpha_{2} m_{2 n}+\alpha_{3}}=+\infty,
$$

then system (5.3) - (5.6) is asymptotically stable, that is any solution $u$ of (5.3) - (5.6) satisfies $E_{S}(u, t) \rightarrow 0$ for $t \rightarrow+\infty$.

Proof. We have only to show that the second condition of (5.14) implies the second condition of (4.9), namely in this case

$$
\sum_{n=0}^{\infty} \ln \frac{\alpha_{1} M_{2 n} m_{2 n}+\alpha_{2} m_{2 n}+\alpha_{3}}{\alpha_{1} M_{2 n} m_{2 n}+\alpha_{2} m_{2 n}+\alpha_{3}+m_{2 n}\left(T_{2 n}-\bar{T}\right)}=-\infty .
$$

Now, observe that

$$
\ln \frac{\alpha_{1} M_{2 n} m_{2 n}+\alpha_{2} m_{2 n}+\alpha_{3}}{\alpha_{1} M_{2 n} m_{2 n}+\alpha_{2} m_{2 n}+\alpha_{3}+m_{2 n}\left(T_{2 n}-\bar{T}\right)}=-\ln \left(1+\frac{m_{2 n}\left(T_{2 n}-\bar{T}\right)}{\alpha_{1} M_{2 n} m_{2 n}+\alpha_{2} m_{2 n}+\alpha_{3}}\right),
$$

then we can conclude arguing as in the proof of Theorem 3.9.

Remark 5.3 If $T_{2 n}=T^{*}$ and $M_{2 n}=M^{*}$ as well as $m_{2 n}=m^{*}$, then (5.14) holds if the easily checked condition

$$
\sum_{n=0}^{\infty} M_{2 n+1} T_{2 n+1}<+\infty
$$

holds (since the second condition of (5.14) automatically holds).

Similarly using Theorem 4.5, we directly can state the

Theorem 5.4 Assume that $\left.\left.i_{w}\right), i i_{w}\right)$ and (3.30) hold, that $T_{2 n}=T^{*}$, for all $n \in \mathbb{N}$, with $T^{*}$ satisfying $T^{*} \geq \tau$ and $T^{*}>\bar{T}$ with $\bar{T}$ from Proposition 5.1 Moreover, assume $T_{2 n+1}=\tilde{T}$, for all $n \in \mathbb{N}$ with $\tilde{T} \leq \tau$. If

$$
\sup _{n \in \mathbb{N}}\left[e^{C M_{2 n+1} \tilde{T}}\left(\hat{d}_{n}+1\right)-1\right]<1,
$$

where

$$
\hat{d}_{n}=\frac{\alpha_{1} M_{2 n} m_{2 n}+\alpha_{2} m_{2 n}+\alpha_{3}}{\alpha_{1} M_{2 n} m_{2 n}+\alpha_{2} m_{2 n}+\alpha_{3}+m_{2 n}\left(T_{2 n}-\bar{T}\right)},
$$

then there exist two positive constants $\hat{\gamma}, \hat{\mu}$ such that

$$
E_{S}(t) \leq \hat{\gamma} e^{-\hat{\mu} t} E_{S}(0), \quad t>0,
$$

for any solution of problem (5.3) - (5.6).

As in the abstract setting (see Remarks 4.4 and 4.6), explicit conditions on $b_{1}, b_{2}$ and $T_{2 n}$ can be found in order to get exponential decay. 


\subsection{The wave equation with internal delayed/undelayed feedbacks}

Here we consider the wave equation with local internal damping and internal delay. More precisely, let $\Omega \subset \mathbb{R}^{n}$ be an open bounded domain with a boundary $\partial \Omega$ of class $C^{2}$. Denoting by $m$, as before, the standard multiplier $m(x)=x-x_{0}, x_{0} \in \mathbb{R}^{n}$, let $\omega_{1}$ be the intersection of $\Omega$ with an open neighborhood of the subset of $\partial \Omega$

$$
\Gamma_{0}=\{x \in \partial \Omega: m(x) \cdot \nu(x)>0\} .
$$

Let us consider the initial boundary value problem

$$
\begin{aligned}
& u_{t t}(x, t)-\Delta u(x, t)+b_{1}(t) \chi_{\omega_{1}} u_{t}(x, t)+b_{2}(t) \chi_{\omega_{2}} u_{t}(x, t-\tau)=0 \text { in } \Omega \times(0,+\infty) \\
& u(x, t)=0 \text { on } \partial \Omega \times(0,+\infty) \\
& u(x, 0)=u_{0}(x) \text { and } u_{t}(x, 0)=u_{1}(x) \text { in } \Omega
\end{aligned}
$$

with initial data $\left(u_{0}, u_{1}\right) \in H_{0}^{1}(\Omega) \times L^{2}(\Omega)$ and $b_{1}, b_{2}$ in $L^{\infty}(0,+\infty)$ such that

$$
b_{1}(t) b_{2}(t)=0, \quad \forall t>0 .
$$

Moreover, we assume $i_{w}$ ) and $\left.i i_{w}\right)$.

This problem enters into our previous framework, if we take $H=L^{2}(\Omega)$ and the operator $A$ defined by

$$
A: \mathcal{D}(A) \rightarrow H: u \rightarrow-\Delta u
$$

where $\mathcal{D}(A)=H_{0}^{1}(\Omega) \cap H^{2}(\Omega)$.

The operator $A$ is a self-adjoint and positive operator with a compact inverse in $H$ and is such that $V=\mathcal{D}\left(A^{1 / 2}\right)=H_{0}^{1}(\Omega)$. We then define $U_{i}=L^{2}\left(\omega_{i}\right)$ and the operators $B_{i}, i=1,2$, as

$$
B_{i}: U_{i} \rightarrow H: \quad v \rightarrow \sqrt{b_{i}} \tilde{v} \chi_{\omega_{i}}
$$

where $\tilde{v} \in L^{2}(\Omega)$ is the extension of $v$ by zero outside $\omega_{i}$. It is easy to verify that

$$
B_{i}^{*}(\varphi)=\sqrt{b_{i}} \varphi_{\omega_{i}} \text { for } \varphi \in H,
$$

and thus (3.1) holds with $W_{i}=L^{2}\left(\omega_{i}\right)$, while $B_{i} B_{i}^{*}(\varphi)=b_{i} \varphi \chi_{\omega_{i}}$, for $\varphi \in H$ and $i=1$, 2. This shows that problem (5.18)-(5.20) enters in the abstract framework (1.1)-(1.2). Moreover, $\left.i_{w}\right)$ and $i i_{w}$ ) easily imply $i$ ) and $i i$ ) of sect. 3. Therefore we can restate Proposition 3.1. Now, the energy functional is

$$
E(t)=\frac{1}{2} \int_{\Omega}\left\{u_{t}^{2}(x, t)+|\nabla u(x, t)|^{2}\right\} d x+\frac{\xi}{2} \int_{t-\tau}^{t}\left|b_{2}(s+\tau)\right| \int_{\omega} u_{t}^{2}(x, s) d x d s .
$$

Consider now the conservative system

$$
\begin{aligned}
& w_{t t}(x, t)-\Delta w(x, t)=0 \quad \text { in } \quad \Omega \times(0,+\infty) \\
& w(x, t)=0 \text { on } \partial \Omega \times(0,+\infty) \\
& w(x, 0)=w_{0}(x) \text { and } w_{t}(x, 0)=w_{1}(x) \text { in } \Omega
\end{aligned}
$$


with $\left(w_{0}, w_{1}\right) \in H_{0}^{1}(\Omega) \times L^{2}(\Omega)$. It is well-known that an observability inequality holds (see e.g. [3, 10, 11, 13, 14, 15, 16, 23]): There exists a time $\bar{T}>0$ such that for every time $T>\bar{T}$ there is a constant $c$, depending on $T$ but independent of the initial data, such that

$$
E_{S}(0) \leq c \int_{0}^{T} \int_{\omega_{1}} w_{t}^{2}(x, s) d x d s
$$

for every weak solution of problem (5.23) - (5.25).

In the case $\omega_{1}=\omega_{2}$ we can apply the results of section 3.1. Therefore we can restate Proposition 3.2 and Theorems 3.3 and 3.4 .

Proposition 5.5 Assume $\left.\omega_{1}=\omega_{2}, \mathrm{i}_{w}\right), \mathrm{ii}_{w}$ ) and (3.2) are satisfied. Moreover, we assume that the observability inequality (5.26) holds for every time $T>\bar{T}$ and that, denoting $T^{*}:=\inf _{n}\left\{T_{2 n}\right\}$, the assumption (3.11) holds. Then, for any solution of system (5.18) - (5.20) we have

$$
E\left(t_{2 n+1}\right) \leq c_{n} E\left(t_{2 n}\right), \quad \forall n \in \mathbb{N},
$$

where

$$
c_{n}=\frac{4 c\left(1+4 T_{2 n}^{2} M_{2 n}^{2}\right)}{m_{2 n}+4 c\left(1+4 T_{2 n}^{2} M_{2 n}^{2}\right)},
$$

$c$ being the observability constant in (5.26) corresponding to the time $T^{*}$ and $C$ the constant in the norm embedding (3.1) between $W$ and $H$.

Theorem 5.6 Under the assumptions of Proposition 5.5. if

$$
\sum_{n=0}^{\infty} M_{2 n+1} T_{2 n+1}<+\infty \text { and } \sum_{n=0}^{\infty} \frac{m_{2 n}}{1+4 T_{2 n}^{2} M_{2 n}^{2}}=+\infty
$$

then system (5.18) - (5.20) is asymptotically stable, that is any solution $u$ of (5.18) - (5.20) satisfies $E(u, t) \rightarrow 0$ for $t \rightarrow+\infty$.

Theorem 5.7 Assume $\left.\left.\omega_{1}=\omega_{2}, \mathrm{i}_{w}\right), \mathrm{i}_{w}\right)$ and (3.2) are satisfied. Assume also that the observability inequality (5.26) holds for every time $T>\bar{T}$ and that

$$
T_{2 n}=T^{*} \quad \forall n \in \mathbb{N},
$$

with $T^{*}$ satisfying (3.11), and

$$
T_{2 n+1}=\tilde{T} \quad \forall n \in \mathbb{N} .
$$

Moreover, assume that

$$
\sup _{n \in \mathbb{N}} e^{\left(\xi+\frac{1}{\xi}\right) M_{2 n+1} \tilde{T}} c_{n}=d<1
$$

where $c_{n}$ is as in (5.28). Then, there exist two positive constants $\gamma, \mu$ such that

$$
E(t) \leq \gamma e^{-\mu t} E(0), \quad t>0,
$$

for any solution of problem (5.18) - (5.20).

Remark 5.8 The case $\omega_{1}=\omega_{2}$ was already considered in [19]. Note that we significantly improve previous stability results (cfr. Theorems 4.3 and 4.4 in [19]). 
Under the restriction (3.30) we can obtain stability results also in the case $\omega_{1} \neq \omega_{2}$. Note that the sets $\omega_{1}$ and $\omega_{2}$ may also have empty intersection.

Indeed, we can restate Proposition 3.8 and Theorem 3.9 for our concrete model.

Proposition 5.9 Assume $\left.\mathrm{i}_{w}\right), \mathrm{ii}_{w}$ ) and (3.30). Moreover, we assume that the observability inequality (5.26) holds for every time $T>\bar{T}$ and that, denoting $T^{*}:=\inf _{n}\left\{T_{2 n}\right\}$, (3.11) is satisfied. Then, for any solution of system (5.18) - (5.20) we have

$$
E_{S}\left(t_{2 n+1}\right) \leq \hat{c}_{n} E_{S}\left(t_{2 n}\right), \quad \forall n \in \mathbb{N}
$$

where

$$
\hat{c}_{n}=\frac{2 c\left(1+4 T_{2 n}^{2} M_{2 n}^{2}\right)}{m_{2 n}+2 c\left(1+4 T_{2 n}^{2} M_{2 n}^{2}\right)},
$$

c being the observability constant in (5.26) corresponding to the time $T^{*}$.

Theorem 5.10 Under the assumptions of Proposition 5.9, if (3.21) holds, then system (5.18) (5.20) is asymptotically stable, that is any solution $u$ of (5.18) - (5.20) satisfies $E_{S}(u, t) \rightarrow 0$ for $t \rightarrow+\infty$.

Under more restrictive assumption also an exponential stability estimate holds.

Theorem 5.11 Assume $\mathrm{i}_{w}$ ), $\mathrm{ii}_{w}$ ) and (3.30). Assume also that the observability inequality (5.26) holds for every time $T>\bar{T}$ and that

$$
T_{2 n}=T^{*} \quad \forall n \in \mathbb{N},
$$

with $T^{*}$ satisfying (3.11), and

$$
T_{2 n+1}=\tilde{T} \quad \forall n \in \mathbb{N},
$$

with $\tilde{T} \leq \tau$.

Moreover, assume that

$$
\sup _{n \in \mathbb{N}}\left[e^{M_{2 n+1} \tilde{T}}\left(\hat{c}_{n}+1\right)-1\right]=\hat{d}<1
$$

where $\hat{c}_{n}$ is as in (5.33). Then, there exist two positive constants $\hat{\gamma}, \hat{\mu}$ such that

$$
E_{S}(t) \leq \hat{\gamma} e^{-\hat{\mu} t} E_{S}(0), \quad t>0,
$$

for any solution of problem (5.18) - (5.20).

Remark 5.12 Note that the case $\omega_{1} \neq \omega_{2}$ is not covered from the abstract setting of [19].

\section{References}

[1] K. Ammari, S. Nicaise and C. Pignotti. Feedback boundary stabilization of wave equations with interior delay. Systems and Control Lett., 59:623-628, 2010.

[2] K. Ammari, S. Nicaise and C. Pignotti. Stabilization by switching time-delay. Asymptot. Anal., 83:263-283, 2013. 
[3] C. Bardos, G. Lebeau and J. Rauch. Sharp sufficient conditions for the observation, control and stabilization of waves from the boundary. SIAM J. Control Optim., 30:1024-1065, 1992.

[4] R. Datko. Not all feedback stabilized hyperbolic systems are robust with respect to small time delays in their feedbacks. SIAM J. Control Optim., 26:697-713, 1988.

[5] R. Datko, J. Lagnese and M. P. Polis. An example on the effect of time delays in boundary feedback stabilization of wave equations. SIAM J. Control Optim., 24:152-156, 1986.

[6] M. Gugat. Boundary feedback stabilization by time delay for one-dimensional wave equations. IMA J. Math. Control Inform., 27:189-203, 2010.

[7] A. Haraux, P. Martinez and J. Vancostenoble. Asymptotic stability for intermittently controlled second-order evolution equations. SIAM J. Control Optim., 43:2089-2108, 2005.

[8] T. Kato. Linear and quasilinear equations of evolution of hyperbolic type. C.I.M.E., II ciclo:125-191, 1976.

[9] T. Kato. Abstract differential equations and nonlinear mixed problems. Lezioni Fermiane. [Fermi Lectures]. Scuola Normale Superiore, Pisa, 1985.

[10] V. Komornik. Exact controllability and stabilization, the multiplier method, volume 36 of RMA. Masson, Paris, 1994.

[11] V. Komornik and P. Loreti. Fourier series in control theory, Springer Monographs in Mathematics, Springer-Verlag, New York, 2005.

[12] V. Komornik and E. Zuazua. A direct method for the boundary stabilization of the wave equation. J. Math. Pures Appl., 69:33-54, 1990.

[13] J. Lagnese. Control of wave processes with distributed control supported on a subregion. SIAM J. Control Optim., 21:68-85, 1983.

[14] I. Lasiecka and R. Triggiani. Uniform exponential decay in a bounded region with $L_{2}\left(0, T ; L_{2}(\Sigma)\right)$-feedback control in the Dirichlet boundary conditions. J. Differential Equations, 66:340-390, 1987.

[15] J. L. Lions. Contrôlabilité exacte, perturbations et stabilisation des systèmes distribués, Tome 1, volume 8 of RMA. Masson, Paris, 1988.

[16] K. Liu. Locally distributed control and damping for the conservative systems. SIAM J. Control and Optim., 35:1574-1590, 1997.

[17] S. Nicaise and C. Pignotti. Stability and instability results of the wave equation with a delay term in the boundary or internal feedbacks. SIAM J. Control Optim., 45:1561-1585, 2006.

[18] S. Nicaise and C. Pignotti. Interior feedback stabilization of wave equations with time dependent delay, Electron. J. Differential Equations, 2011:1-20, 2011.

[19] S. Nicaise and C. Pignotti. Asymptotic stability of second-order evolution equations with intermittent delay. Adv. Differential Equations, 17:879-902, 2012. 
[20] C. Pignotti. A note on stabilization of locally damped wave equations with time delay. Systems Control Lett., 61:92-97, 2012.

[21] M. Tucsnak and G. Weiss. Observation and control for operator semigroups, Birkhäuser Advanced Texts: Basler Lehrbücher. Birkhäuser Verlag, Basel, 2009.

[22] G. Q. Xu, S. P. Yung and L. K. Li. Stabilization of wave systems with input delay in the boundary control. ESAIM: Control Optim. Calc. Var., 12:770-785, 2006.

[23] E. Zuazua. Exponential decay for the semi-linear wave equation with locally distributed damping. Comm. Partial Differential Equations, 15:205-235, 1990.

E-mail address,

Serge Nicaise: snicaise@univ-valenciennes.fr

Cristina Pignotti: pignotti@univaq.it 